\title{
Spodoptera exigua (J. E. Smith) (Lepidoptera: Noctuidae) como plaga de albahaca en Morelos, México
}

\author{
Spodoptera exigua (J. E. Smith) (Lepidoptera: Noctuidae) as a pest of basil in Morelos, Mexico
}

\author{
Juan Francisco Aguirre-Cadena ${ }^{1}$ (D) , Dagoberto Guillen-Sánchez ${ }^{2}$ (D) ,
} Nidia Bélgica Pérez-De la $\mathrm{O}^{* 3}$ (D) , Alfredo Isaac Brindis-Santos ${ }^{1}$

${ }^{1}$ Facultad de Ciencias Agrícolas, Campus IV, Universidad Autónoma de Chiapas, Entronque Carretera Costera y Pueblo de Huehuetán, Huehuetán 30660, Chiapas, México.

${ }^{2}$ Facultad de Ciencias Agropecuarias, Universidad Autónoma del Estado de Morelos, Av. Universidad 1001, Col. Chamilpa, Cuernavaca 62209, Morelos, México. ${ }^{3}$ Posgrado en Fitosanidad - Entomología y Acarología, Colegio de Postgraduados, Carretera México-Texcoco km 36.5, 56230 Estado de México, México.

*Autor para correspondencia: perez.nidia@colpos.mx

Fecha de recepción:

1 de agosto de 2021

Fecha de aceptación:

19 de noviembre de 2021

\section{Disponible en línea:}

21 de diciembre de 2021

Este es un artículo en acceso abierto que se distribuye de acuerdo a los términos de la licencia Creative Commons.

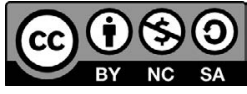

Reconocimiento-

NoComercia-

CompartirIgual 4.0

Internacional

\section{RESUMEN}

La albahaca, Ocimum basilicum L., es una de las principales hierbas aromáticas que se cultivan en México, utilizada como condimento alimentario y valorada por poseer aceites esenciales. En 2019, se registró la presencia explosiva y temporal de un lepidóptero defoliador en Morelos, México; ante el desconocimiento de la identidad taxonómica, se procedió a realizar varias colectas para su determinación específica. Todos los especímenes fueron identificados como Spodoptera exigua (J. E. Smith) (Lepidoptera: Noctuidae), un lepidóptero polífago de distribución amplia en México, pero sin registro formal en el cultivo en esta región del país. Las larvas causaron severo daño foliar y depositaron heces fecales sobre el follaje, aspectos que afectan el rendimiento y calidad de la albahaca de la región.

\section{palabras Clave}

Hierbas aromáticas, plaga polífaga, rendimiento.

\section{ABSTRACT}

Basil, Ocimum basilicum L., is one of the main aromatic herbs grown in Mexico. It is used as a food condiment and valued for having essential oils. In 2019, explosive and temporary damage caused by a lepidopteran defoliator was registered in Morelos, Mexico. As the taxonomic identity of this defoliator was unknown, several collections were carried out for its specific determination. All specimens were identified as Spodoptera exigua (J. E. Smith) (Lepidoptera: Noctuidae), a polyphagous lepidopteran of widely distributed in Mexico, but with no formal record on basil in this region of the country. The larvae caused severe foliar damage and deposited feces on the foliage, aspects that affect the yield and quality of basil in the region.

\section{KEYWORDS}

Aromatic herbs, polyphagous pest, yield. 


\section{INTRODUCCIÓN}

México cuenta con zonas con potencial alto para la producción de hierbas finas, pues su creciente demanda las ha convertido en productos con un nicho de mercado rentable y en expansión; además, representan una alternativa económica a los cultivos tradicionales (Juárez-Rosete et al. 2013). La albahaca, Ocimum basilicum L., es importante dentro de las plantas aromáticas debido a su uso como condimento, además de poseer aceites esenciales que emanan un agradable aroma y sabor (Reyes et al. 2014). Originaria de la India, es empleada como especia y condimento alimentario; asimismo, es una de las plantas aromáticas más apetecidas en el ámbito culinario, por tener un sabor dulce y fragante (Colorado et al. 2013). Igualmente es muy utilizada en medicina tradicional, para curar afecciones gastrointestinales (diarreas, parasitismo), respiratorias (bronquitis, tos), dolor de oídos y reumatismo (Sánchez et al. 2000). Actualmente, ocupa el tercer lugar en superficie sembrada de plantas aromáticas en el país (SIAP 2020). Sin embargo, la investigación en el proceso productivo del cultivo se ha centrado en el norte del país (Reyes et al. 2014; Valenzuela-Escoboza et al. 2019), por lo que se desconoce qué sucede en otras regiones.

\section{Materiales y Métodos}

Se recibieron comentarios de la presencia de un lepidóptero defoliador que, de manera repentina y masiva, afectó varias naves en producción de albahaca en dos localidades del municipio de Mazatepec, Morelos, México (Mazatepec y Cuauchichinola). Al respecto, en Mazatepec (18.7235, -99.3560), con fechas 3/II/2019, 19/IV/2019 y 3/V/2019, y en Cuachichinola (18.6416, -99.3685), con fechas 16/VI/2021 y 19/ $\mathrm{VI} / 2021$, se realizaron colectas, con la finalidad de identificar taxonómicamente a este defoliador. Larvas en distintos instares fueron colectadas manualmente y transportadas a laboratorio, dentro de recipientes de plástico con tela de organza para facilitar el intercambio gaseoso; de manera continua, fue proporcionado follaje de albahaca para su alimentación. Asimismo, se mantuvo a las pupas de forma individual hasta la emergencia de adultos. Las larvas fueron preservadas en alcohol a 70 por ciento y fueron determinadas con las claves y características morfológicas reportadas por Passoa (1991 y 2014), mientras que adultos fueron corroborados con lo reportado por Oliver y Chapin (1981) y Brambila (2013).

\section{Resultados y Discusión}

Todos los especímenes estudiados fueron determinados como Spodoptera exigua (J. E. Smith) (Lepidoptera: Noctuidae), una especie conocida por su polifagia (Barrientos-Gutiérrez et al. 2013; Camarena 2009; González-Maldonado et al. 2015; Perzabal-Ramos et al. 2018), pero apenas mencionada como problema fitosanitario en albahaca (Valenzuela-Escoboza et al. 2019). Los daños observados en el cultivo fueron defoliación y presencia de heces (figura 1); la defoliación demerita la calidad y rendimiento del cultivo. En caso de exportación, los criterios en suciedad dependen del tamaño de los insectos y la distribución de ésta en la muestra (FDA 2005), por lo que, en el consumo en fresco de la albahaca, las heces pueden restar calidad. Por el momento, no existen productos fitosanitarios aprobados para el manejo de poblaciones de insectos plaga en el cultivo (CICOPLAFEST 1991); sin embargo, la correcta identificación de las especies que afectan el rendimiento y calidad de los cultivos agrícolas es

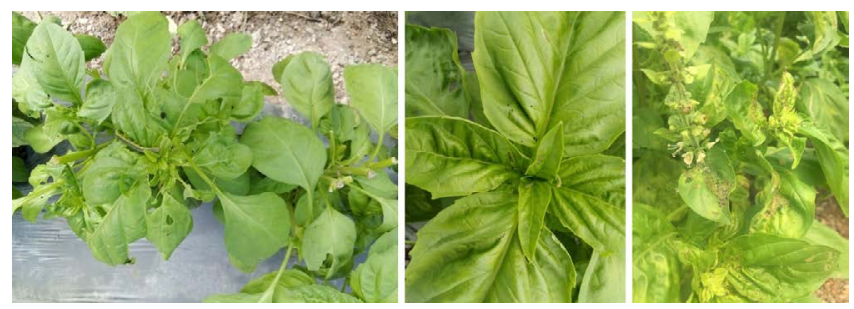

Figura 1. Daños asociados a Spodoptera exigua (J. E. Smith) (Lepidoptera: Noctuidae) en albahaca.

fundamental para seleccionar técnicas apropiadas de monitoreo (por ejemplo, feromona sexual y trampas) y control (aplicación de entomopatógenos y/o enemigos naturales). 


\section{Literatura Citada}

Barrientos-Gutiérrez JE, Huerta-de la Peña A, EscobedoGarrido JS y López-Olguín JF. 2013. Manejo convencional de Spodoptera exigua en cultivos del municipio de Los Reyes de Juárez, Puebla. Revista Mexicana de Ciencias Agrícolas 4: 1197-1208.

Brambila J. 2013. Identification notes for Spodoptera litura and Spodoptera littoralis (Lepidoptera: Noctuidae) and some native Spodoptera moths. Folleto Técnico. USDA-Animal and Plant Health Inspection ServicePlant Protection and Quarantine. Washington, D.C.

Camarena G. 2009. Señales de la interacción planta insecto. Revista Chapingo. Serie Ciencias Forestales y del Ambiente 15: 81-85.

[CICOPLAFEST] Comisión Intersecretarial para el Control del Proceso y Uso de Plaguicidas, Fertilizantes y Sustancias Tóxicas. [internet]. 1991. Catálogo Oficial de Plaguicidas. México, D. F. [citado 2021 enero 20]. Disponible en: https://biblioteca.semarnat.gob.mx/ janium/Documentos/Ciga/agenda/PP03/catalogo.pdf

Colorado F, Montañez I, Bolaños C y Rey J. 2013. Crecimiento y desarrollo de albahaca (Ocimum basilicum L.) bajo cubierta en la sabana de Bogotá. Revista U.D.C.A Actualidad \& Divulgación Científica 16: 121129. https://doi.org/10.31910/rudca.v16.n1.2013.866

[FDA] United States Fodd and Drug Administration. [internet]. 2005. Food Defect Levels Handbook. [citado 2021 enero 30]. Disponible en: https://www. fda.gov/food/ingredients-additives-gras-packaging-guidance-documents-regulatory-information/ food-defect-levels-handbook.

González-Maldonado MB, Gurrola-Reyes JN y ChaírezHernández I. 2015. Productos biológicos para el control de Spodoptera frugiperda (Lepidoptera: Noctuidae). Revista Colombiana de Entomología 41: 200-204.

Juárez-Rosete CR, Aguilar-Castillo JA, Juárez-Rosete ME, Bugarín-Montoya R, Juárez-López P y CruzCrespo E. 2013. Hierbas aromáticas y medicinales en México: tradición e innovación. Revista Bio Ciencias 2: 119-129.

Oliver AD y Chapin JB. 1981. Biology and illustrated key for the identification of twenty species of economically important noctuid pests. LSU Agricultural Experiment Station Reports 260: 1-26.

Passoa S. 1991. Color identification of economically important Spodoptera larvae in Honduras (Lepidoptera:
Noctuidae). Insecta Mundi 5: 185-196.

Passoa SC. 2014. LepIntercept-An Identification Resource for Intercepted Lepidoptera Larvae. Identification Technology Program-USDA. Fort Collins, Estados Unidos.

Perzabal-Ramos M, Sandoval-Castro E, Díaz-Ruíz R, Huerta-de la Peña A, Figueroa-Brito R y Bahena-Juárez F. 2018. Respuesta de espinaca y de Spodoptera exigua a fertilización orgánica y mineral. Revista Mexicana de Ciencias Agrícolas 9: 723-735. https://doi.org/10.29312/ remexca.v9i4.1390

Reyes JJ, Murillo B, Nieto A, Troyo E, Reynaldo IM, Rueda EO y Cuervo JL. 2014. Crecimiento y desarrollo de variedades de albahaca (Ocimum basilicum L.) en condiciones de salinidad. Tierra Latinoamericana 32: 35-45.

Sánchez E, Leal IM, Fuentes L y Rodríguez CA. 2000. Estudio farmacognóstico de Ocimum basilicum L. (albahaca blanca). Revista Cubana de Farmacia 34: 187-95.

[SIAP] Servicio de Información Agroalimentaria y Pesquera. [internet]. 2020. Producción Anual Agrícola 2020. [citado 2021 enero 10]. Disponible en: https:// nube.siap.gob.mx/cierreagricola/2020.

Valenzuela-Escoboza FA, Palacios-Torres RE, ValdezCarrasco JM, Santillán-Galicia MT, Cortez-Moncada E y Ayala-Armenta QA. 2019. Calycomyza hyptidis y sus parasitoides, primer reporte en albahaca, en México. Southwestern Entomologist 44: 695-704. https://doi. org/10.3958/059.044.0301 